\author{
Original Article
}

\title{
Evaluation of Enamel Roughness in Vitro After Orthodontic Bracket Debonding Using Different Methods of Residual Adhesive Removal
}

José Tarcísio Lima Ferreira (iD, Maria Cristina Borsatto (D), Maria Conceição Pereira Saraiva (DD, Mírian Aiko Nakane Matsumoto (iD, Carolina Paes Torres (D), Fabio Lourenço Romano (D)

Department of Pediatrics Dentistry, University of São Paulo Ribeirão Preto, São Paulo, Brazil

Cite this article as: Ferreira JTL, Borsatto MC, Saraiva MCP, Matsumoto MAN, Torres CP, Romano FL. Evaluation of Enamel Roughness in Vitro After Orthodontic Bracket Debonding Using Different Methods of Residual Adhesive Removal. Turk J Orthod 2020; 33(1): 43-51.

\begin{abstract}
Objective: The aim of the present study was to compare different techniques for resin remnant removal (RRR) after orthodontic bracket debonding and to evaluate alterations on the dental enamel caused by these methods. The null hypothesis tested was that there is no difference between RRR techniques in relationship the changes caused on the dental enamel.

Methods: A total of 75 bovine mandibular permanent incisors were used in the study. Brackets were bonded and debonded in each tooth in two experimental regions. Five RRR techniques were used in the experimental groups ( $n=15)$ : Group 1-diamond bur (6-bladed), Group 2-diamond bur (12-bladed), Group 3-diamond bur (30-bladed), Group 4-aluminum oxide sandblasting (AOS), and Group 5-Er:YAG laser. Enamel surface was evaluated using profilometry, and surface roughness analysis was performed at three time intervals: before bracket bonding, after RRR techniques, and after final polishing. Qualitative analyses of the enamel surfaces were performed using scanning electron microscopy.
\end{abstract}

Results: Multiblade burs showed the best results, and the 30-bladed bur created a less irregular enamel surface. AOS caused greater enamel wear, and Er:YAG laser caused more surface irregularity.

Conclusion: The null hypothesis was rejected. The multiblade burs were the least harmful than the other techniques. Enamel surface roughness after using the 30-blade bur was similar to the original enamel. These results indicate that the type of bur tested (30-bladed) can be indicated to remove resin remnants after bracket debonding.

Keywords: Orthodontic debonding, enamel, orthodontic brackets

\section{INTRODUCTION}

Orthodontic brackets are fixated to the enamel for the purpose of providing support to perform orthodontic mechanics. Over the past few years, composites have been used to bond brackets to the enamel. In most cases, bonding is obtained through mechanical retention of both the bonding agent and composite to the micropores created by acid etching on the enamel surface and by the interlocking of the composite in the bracket base mesh $(1,2)$.

Once treatment has been completed, the orthodontic appliance must be removed. After bracket debonding, regardless of the method used, the ideal situation is that all composite used for fixation remains adhered to the enamel, thus protecting the surface against possible fractures $(3,4)$. When the brackets are removed, the resin remnants on the enamel must also be removed. 
Mechanical removal for the remaining composite, after debonding orthodontic brackets, has been shown to be detrimental to the enamel surface (5-8). Studies have assessed composite resin remnant removal (RRR) from the enamel, after bracket debonding, using various methods, including pliers, low- and highspeed drill with burs, manual scrapers, ultrasound, aluminum oxide sandblasting (AOS), and lasers (5-19).

During the bonding and debonding processes using the appropriate technique, it is estimated that $5-20 \mu \mathrm{m}$ of the enamel is lost (20-22). Koprowski et al. (23) assessed the quality of the enamel after the treatment and clean-up procedure of debonding brackets through the use of computed tomography. The results showed that the enamel thickness after the orthodontic treatment had decreased by approximately $125 \mu \mathrm{m}$. Ulusoy (24) expressed that the search for the ideal method, which returns the enamel surface as closely as possible to its original state, is still ongoing. After removing the remnants of bonding material, it is a consensus that prophylaxis must be performed using a low-speed motor with a rubber cup, pumice stone paste, and water to polish the enamel (25).

The aim of the present study was to evaluate and compare, using profilometry and surface roughness, the following: 1) different techniques for removing resin remnants after orthodontic bracket debonding and 2) possible alterations on the enamel caused by these methods. The null hypothesis tested was that there is no difference between RRR techniques and polishing and there is no interaction among them.

\section{METHODS}

The approval letter of the ethics committee was not included because, in accordance with the provisions of Art. 3 Item III and Art. 10 of Law 11,794 of 10/08/2008, experimental protocols that do not involve the use of live animals should not be analyzed.

Sample size was based on the study by Ahrari, considering an effect size of 0.25 , power of 0.80 , and alpha of 0.05 for six groups with a correlation of 0.10 . Sample size was calculated using the $\mathrm{G}$ *Power program for repeated measurements.

A total of 75 bovine mandibular permanent incisors were stored for 1 week in $0.1 \%$ thymol for disinfection. The criteria for selection of teeth were intact crowns and absence of demineralization, cracks, fractures, or stains. The roots were sectioned $(2 \mathrm{~mm}$ below the cement-enamel junction) using a water-cooled double-faced diamond disk (KG Sorensen, 7015, Brazil) mounted on a sectioning machine (Miniton; Struers A/S, Denmark). The buccal face of each crown was fixed onto a glass plate using utility wax. They were then embedded in self-curing acrylic resin using a plastic cylinder (20 mm diameter $\times 2 \mathrm{~cm}$ high) as a mold. Specimens were polished (240-, 600-, and 1200-grit silicon carbide papers) in a polishing machine (Struers, Copenhagen, Denmark) to expose an area of at least $15 \mathrm{~mm}$ long in the cervical-incisal direction. The surface received final polishing (damp felt and 0.3 - and $0.5-\mu \mathrm{m}$ aluminum particle) to obtain an enamel with- out scratches. Buccal faces were randomly divided into three regions, one control and two experimental, and covered with a yellow adhesive polyethylene tape "mask" measuring $70 \mathrm{~mm} \times$ $200 \mathrm{~mm}$. Teeth were stored in distilled water at $37^{\circ} \mathrm{C}$.

Prophylaxis was performed for $10 \mathrm{~s}$ using fluoride-free pumice and water slurry on a rubber cup, followed by washing and drying at the same time. Rubber cups were replaced after five uses. In the two experimental groups, the enamel surfaces were etched (37\% phosphoric acid gel, $15 \mathrm{~s}$ and washed and dried, 15 s) and coated with a layer of adhesive primer (Transbond XT; $3 \mathrm{M}$ Unitek, Monrovia, CA, USA), and 150 standard edgewise metal brackets for lower incisors (base area of $9.78 \mathrm{~mm}^{2}$; Morelli, Sorocaba, SP, Brazil) were bonded with Transbond XT composite resin (3M Unitek). The adhesive was cured for $40 \mathrm{~s}$ on the mesial, distal, incisal, and cervical faces (10 s/each face) at $1 \mathrm{~mm}$ from the bracket base using a halogen light-curing unit XL 1500 (3M ESPE, St. Paul, MN, USA) with a light intensity of $400 \mathrm{~mW} / \mathrm{cm}^{2}$, verified after five activations with a Demetron curing radiometer (Danbury, NH, USA) (Figure 1). Test specimens were stored in distilled water $\left(37^{\circ} \mathrm{C}\right.$ for $\left.24 \mathrm{~h}\right)$, and then all brackets were debonded (JTLF) with curved How pliers no. 110 (3M Unitek).

Five groups ( $n=15)$ were formed according to the RRR technique (Table 1). In each test specimen, three regions were established using a table of random numbers in: 1) control area, 2) enamel after RRR, without final polishing, and 3) enamel after RRR, with final polishing. The polishing was performed for $10 \mathrm{~s}$ using fluoride-free pumice and water slurry on a rubber cup, followed by washing and drying for the same amount of time.

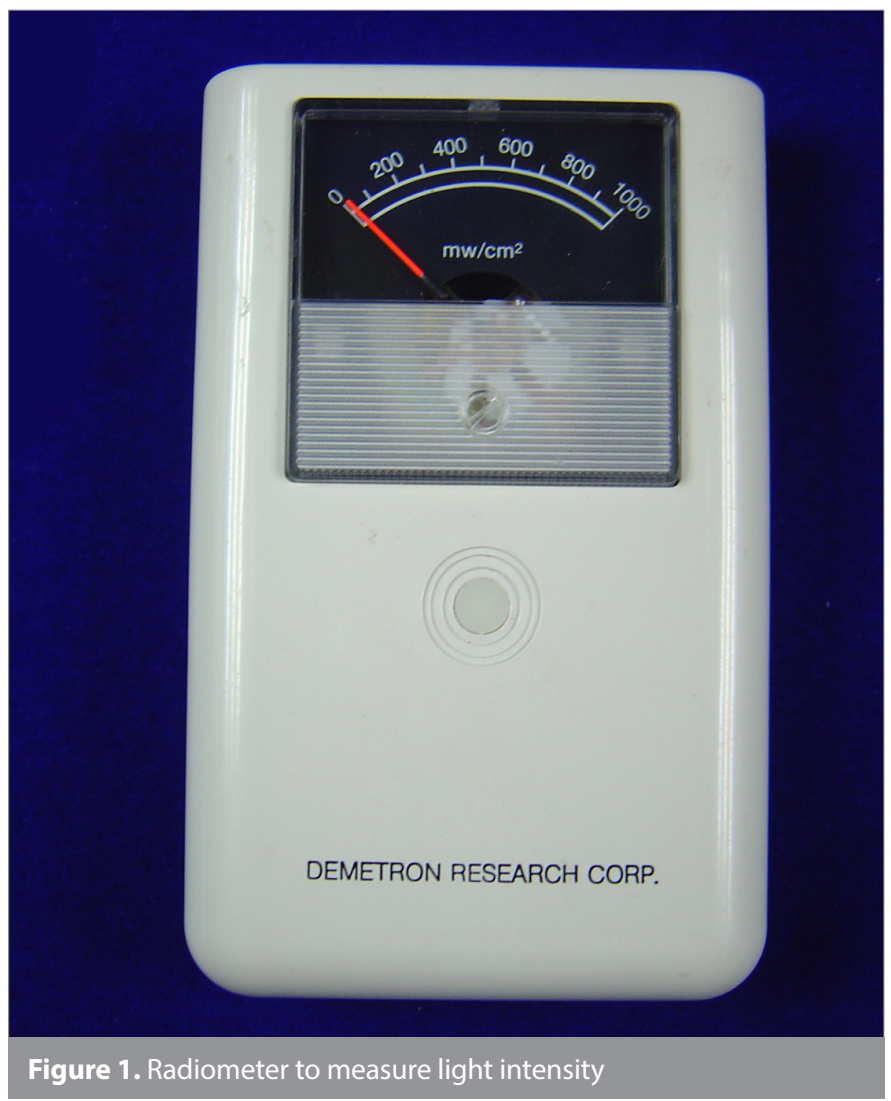




\begin{tabular}{|c|c|c|}
\hline $\begin{array}{l}\text { Group } \\
(n=15)\end{array}$ & $\begin{array}{l}\text { Resin remnant } \\
\text { removal techniques }\end{array}$ & Manufacturer \\
\hline $30 \mathrm{~B}$ & 30-bladed bur & Beavers Dental \\
\hline $12 B$ & 12-bladed bur & Beavers Dental \\
\hline $6 \mathrm{~B}$ & 6-bladed bur & TP Orthodontics \\
\hline AOS & $\begin{array}{l}\text { Aluminum oxide } \\
\text { sandblasting } 50 \mu \mathrm{m}\end{array}$ & VH Equipments \\
\hline Laser & Er:YAG laser & Fotona Medical Lasers \\
\hline
\end{tabular}

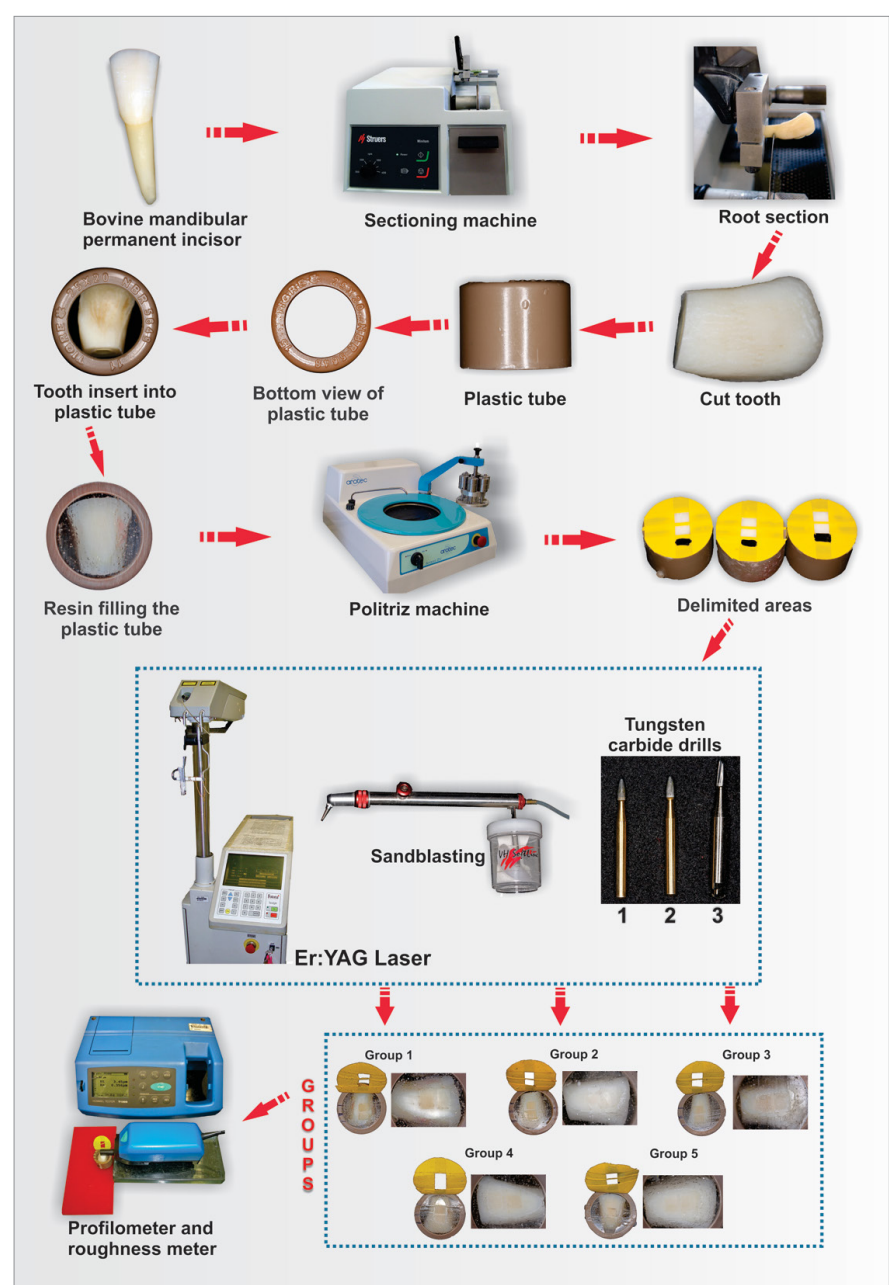

Figure 2. Flowchart of the study steps

The RRR from the enamel after bracket debonding was performed by three techniques: multibladed burs, AOS, and Er:YAG laser. For the multibladed burs, 6-bladed (TP Orthodontics, La Porte, IN, USA) and 12-bladed and 30-bladed (jet carbide burs; Beavers Dental, Morrisburg, ON, Canada) were applied with paintbrush movement, at low speed, on the resin remnants. Each multiblade bur was replaced after five uses.

For AOS, the Microjato Gold Line appliance was used (VH Equipments Medical/Dental and Accessories Ltda., Araraquara, SP, Brazil), with a 50- $\mu \mathrm{m}$ aluminum oxide particle stream, a standardized distance of $10 \mathrm{~mm}$ from the tooth surface, at a $45^{\circ}$ angle for $15 \mathrm{~s}$.
The Er:YAG laser Twin Light (Fotona Medical Lasers, Ljubljana, Slovenia) was used with 200-450 ms pulse duration, $2.94 \mu \mathrm{m}$ wavelength, $500 \mathrm{~mJ}$ maximum pulse energy, and $2-15 \mathrm{~Hz}$ pulse repetition rate. This delivery system has two articulated arms with a sapphire window in non-contact mode and an air-water spray cooling system. Irradiation on the enamel was aided by a guide beam directing laser emission on the area to be irradiated. To avoid overheating during irradiation, the air-water spray was activated, and the water flow was regulated at $1.5 \mathrm{~mL} / \mathrm{min}$. Irradiation was performed using parallel horizontal movements from top to bottom in non-contact mode. It was focused perpendicular to the tooth surface, at a distance of $12 \mathrm{~mm}$ with $260 \mathrm{~mJ}$ pulse energy and $47 \mathrm{~J} / \mathrm{cm}^{2}$ energy density, $3 \mathrm{~Hz}$ pulse repetition rate, and $30 \mathrm{~s}$ irradiation time. Enamel surfaces were irradiated using an automatic custom-designed device (MPC ElQuip, São Carlos, SP, Brazil) that was affixed to the laser handpiece in such a manner that the laser beam was delivered perpendicular to the specimen surface at a constant distance from the target site.

After the RRR procedures, enamel surfaces were re-examined. The five groups were evaluated at three time points: 1 ) after prophylaxis prior to bracket bonding (control), 2) after RRR before final polishing, and 3) after RRR after final polishing.

Profilometry and roughness analyses were performed using Hommel Tester T1000 (Hommelwerke GmbH, Schwenningen, Germany) equipment. For roughness, the parameters were established at $L_{t}$ (assessment length): $1.5 \mathrm{~mm}$ and $L_{c}$ (cut-off): $0.25 \mathrm{~mm}$. In the profilometry measurement, the needle of the device was positioned in the region considered to be the control and then from this point for all regions of the specimens. Three random readings were obtained on each evaluated surface. The baseline was obtained using the arithmetic mean of these three readings.

Two teeth from each group were randomly selected for analysis using scanning electron microscopy (SEM) by the $\mathrm{EVO} 50^{\circledR}$ appliance (Carl Zeiss SMT, Gottingen, Germany) operated at $20.00 \mathrm{kV}$.

Figure 2 represents the flowchart of the methodology used in the present study.

\section{Statistical Analysis}

All analyses were performed using the SAS 9.4 (PROCMIXED) statistical program using an alpha of 0.05 . A generalized linear mixed model was used in the analysis for repeated measures. It took into consideration the dependence of data within each tooth specimen. Interaction between type of treatment and polishing was tested. Tukey-Kramer post-test was used for comparison of the adjusted means for each treatment and polishing condition.

\section{RESULTS}

\section{Profilometry Analysis}

The analysis of variance for mixed models for profilometry showed a statistically significant difference between the RRR techniques $(<0.0001)$ and the final polishing type $(0.0049)$. More- 


\begin{tabular}{|c|c|c|c|c|}
\hline \multirow[b]{2}{*}{ Groups } & \multicolumn{2}{|c|}{ Polishing } & \multirow[b]{2}{*}{ Dif $(95 \% \mathrm{CI}) *$} & \multirow[b]{2}{*}{$\mathbf{p}^{* *}$} \\
\hline & Yes & No & & \\
\hline Blade-30 & 7.96 & 5.20 & 2.75 (-4.51 to 10.36$)$ & 0.9675 \\
\hline Blade-12 & 11.39 & 8.62 & 2.77 ( -4.50 to 10.05$)$ & 0.9666 \\
\hline Blade- 6 & 11.66 & 12.10 & -0.43 ( -7.71 to 6.84$)$ & 0.9999 \\
\hline AOS & 29.61 & 32.05 & $-2.44(-9.71$ to 4.83$)$ & 0.9855 \\
\hline Laser & 24.67 & 12.86 & 11.80 (4.53 to 19.08$)$ & $<0.0001$ \\
\hline
\end{tabular}

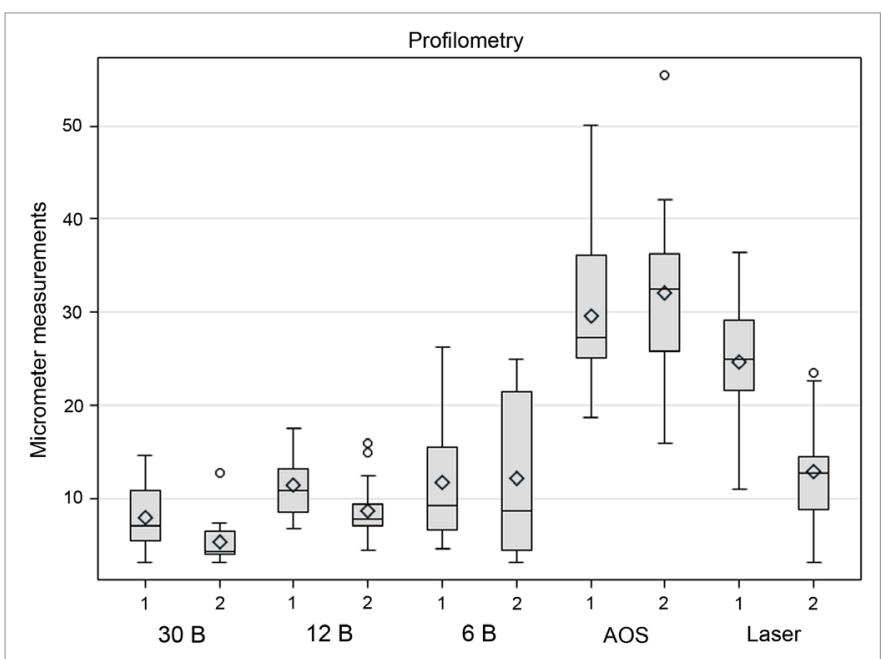

Figure 3. Box-plot showing distribution for profilometry for the different residual removal resin techniques with (number 2 ) and without polishing (number 1). Diamonds represent means only for reference

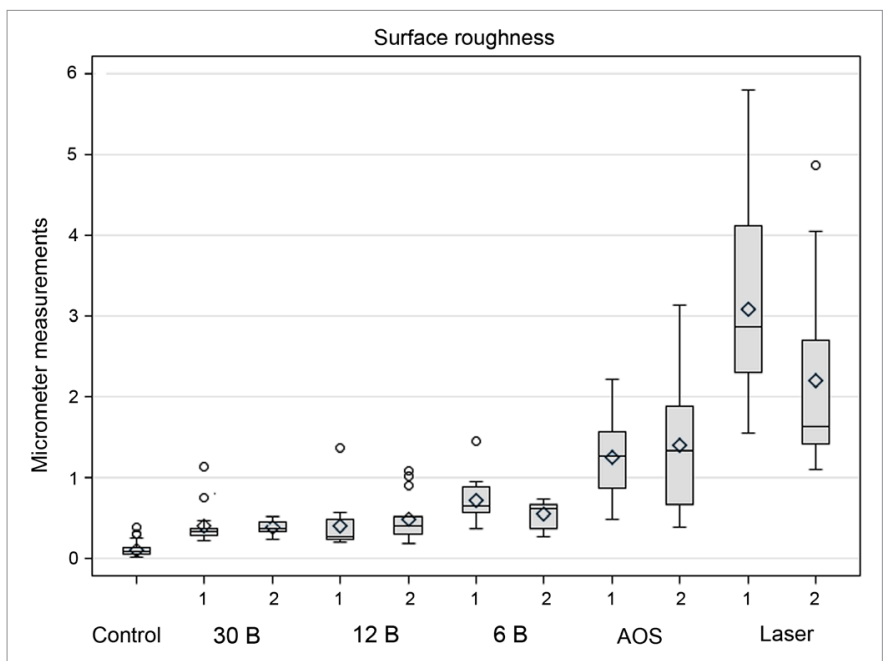

Figure 4. Box-plot showing distribution for surface roughness for the different residual removal resin techniques with (number 2) and without polishing (number 1). Diamonds represent means only for reference

over, there was an interaction between the techniques and polishing (0.0002). This interaction means that polishing interfered with the performance of the techniques, but this interaction only occurred for laser therapy (Table 2). Profilometry means for laser technique was lower $(\mathrm{p}<0.0001)$ for no polishing $(12.86 \mu \mathrm{m})$ and then the one observed for polishing $(24,67 \mu \mathrm{m})$.

Comparison between profilometry means of the RRR techniques can be better illustrated through box-plots in Figure 3 (no. 1 -without polishing and no. 2-with polishing) and in Table 3 in which adjusted mean differences and $95 \%$ confidence intervals are described with indication of those differences that were statistically significant $(p<0.05)$. Table 3 shows a matrix with differences between RRR techniques among polishing in the upper part of the matrix and among non-polishing in the lower part.

There was no observed difference among bladed burs, with or without polishing. Independent of polishing, all bladed burs were statistically different from AOS. The laser was statistically different from all bladed burs in the absence of polishing, and it was not different from 6- to 12-bladed burs when polishing was performed. No difference between the laser and AOS could be observed in the absence of polishing; the two techniques were different when polishing was performed.

\section{Surface Roughness Analysis}

Analysis of variance showed that both techniques $(<0.001)$ and polishing (0.0446) interfered in the final roughness, with significant interaction between technique and polishing (0.0006). The mean comparison revealed that the interaction was due to the differences in polishing only for laser treatment. Polishing did not affect any other treatment.

Box-plots depicting roughness distribution and polishing status are shown in Figure 4 (no. 1-without polishing and no. 2-with polishing). There was no statistical difference between the multiblade burs, regardless of whether final polishing was performed or not (Table 4). All other comparisons between AOS and laser and blades were statistically significant, except for difference between AOS and blade-6 among the samples without polishing (Table 5).

\section{SEM Evaluation}

SEM micrographs obtained from each method of RRR (Figure 5-9) showed that after polishing, the surface on which the AOS was used showed fewer irregularities (Figure 8). The 6-, 12-, and 30-bladed tungsten carbide burs showed surface irregularities even when final polishing was performed (Figure 5-7).

SEM micrographs obtained using AOS and laser showed that both techniques caused very irregular enamel surfaces (Figure $8,9)$.

\section{DISCUSSION}

With the emergence of new materials and techniques in dentistry, research has been conducted to evaluate the possibility of their use in the dental clinic. Thus, orthodontics has been searching for new technologies to RRR adhered to the enamel after debonding procedures. Among the most used techniques, multiblade burs are the most common $(11,26,27)$. Various bur shapes and different blade types are available for clinical use. 


\begin{tabular}{|c|c|c|c|c|c|c|}
\hline & & \multicolumn{5}{|c|}{ Polishing } \\
\hline & & Blade-30 & Blade-12 & Blade-6 & AOS & Laser \\
\hline \multirow{3}{*}{ No polishing } & Blade-12 & $3.4(-3.84$ to 10.70$)$ & 1 & $3.48(-3.79$ to 10.76$)$ & $-23.43^{*}(-30.70$ to -16.15$)$ & $-4.24(11.51$ to -3.03$)$ \\
\hline & Blade-6 & 3.70 (3.57 to 10.98$)$ & $0.27(-7.00$ to 7.55$)$ & 1 & $-19.95^{*}(-27.22$ to -12.67$)$ & $0.76(-6.5$ to 18.04$)$ \\
\hline & Laser & $16.71^{*}(9.43$ to 23.98$)$ & $13.28^{*}(6.00$ to 20.55$)$ & $13.0^{*}$ (5.73 to 20.28$)$ & $4.94(-2.33$ to 12.22$)$ & 1 \\
\hline
\end{tabular}
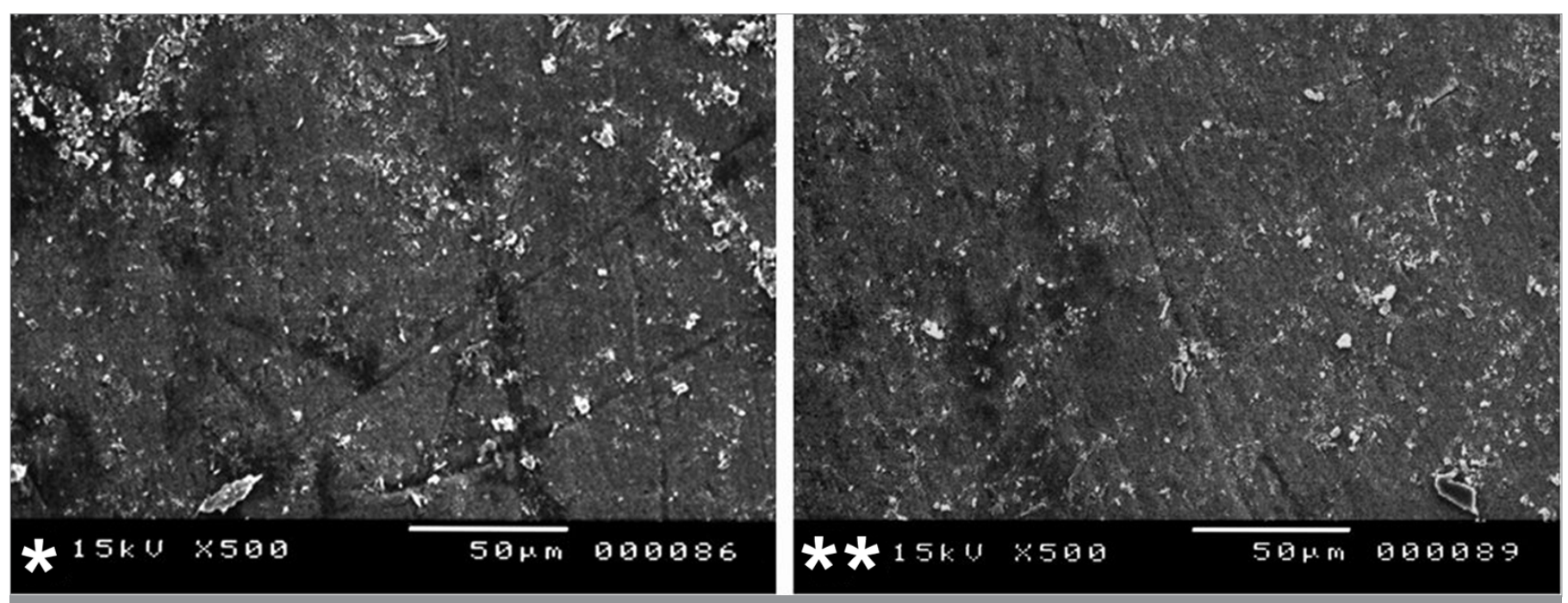

Figure 5. SEM micrograph of enamel surface whose resin remnant was removed by 30 -blade tungsten carbide bur: * without polishing and ${ }^{* *}$ with polishing (500X magnification)

Table 4. Profilometry means and difference $(\mu \mathrm{m})$ for polishing among each removal technique of resin remnants

\begin{tabular}{|c|c|c|c|c|}
\hline \multirow[b]{2}{*}{ Groups } & \multicolumn{2}{|c|}{ Polishing } & \multirow[b]{2}{*}{ Dif $(95 \% \mathrm{CI}) *$} & \multirow[b]{2}{*}{$\mathbf{p}^{* *}$} \\
\hline & Yes & No & & \\
\hline Control & 0.11 & & 0.01 (0.01 to 0.21$)$ & \\
\hline Blade-30 & 0.41 & 0.38 & 0.02 ( -0.58 to 0.62$)$ & 0.9999 \\
\hline Blade-12 & 0.40 & 0.49 & $-0.08(-0.68$ to 0.51$)$ & 0.9999 \\
\hline Blade- 6 & 0.72 & 0.55 & $0.17(-0.43$ to 0.76$)$ & 0.9978 \\
\hline AOS & 1.24 & 1.40 & 0.15 ( -0.75 to 0.44$)$ & 0.9990 \\
\hline Laser & 3.09 & 2.21 & 0.88 (0.28 to 1.48$)$ & 0.0002 \\
\hline
\end{tabular}

Thus, 6-, 12-, and 30-bladed burs were evaluated. Moreover, AOS with 50- $\mu \mathrm{m}$ particles and Er:YAG laser were used to remove the resin remnant after bracket debonding. All techniques were evaluated with and without final polishing. Therefore, this investigation aimed to identify which technique proposed here causes the least amount of alteration in the enamel surface during the RRR procedures. Thus, samples were evaluated and compared using profilometry, whose analysis was obtained in depth, and roughness, whose analysis was superficial.

The average values obtained in the profilometry analysis, considering whether or not final polishing, showed that only the laser technique presented a statistically significant difference. The higher level of depth recorded with the AOS and laser techniques represented a higher loss of enamel. Although polishing was not significant between multiblade burs and AOS, polishing tended to decrease the value found in the profilometry when using different techniques, with the exception of treatments with 6-bladed burs and AOS, which showed higher numeric values after final polishing.

The AOS is dispersed, and therefore, a wide surface region is reached. It is different from the laser, in which the beam is emitted unidirectionally. A size of $50-\mu \mathrm{m}$ particle may have influenced dispersion and thus influenced the results and the greater depth found in the profilometer values when using the technique. Duration of AOS for RRR, although not observed in the present study, was measured by SEM during the time intervals of $5,15,30,45$, and $60 \mathrm{~s}$ and did not cause differences on the enamel surface, as shown by Sargison et al. (15). In contrast, Mhatre et al. (16) compared the RRR with carbide burs and AOS and found a significant difference between them. These authors found lower values in the profilometry, without a show of application time interval and particle size. Thus, larger oxide particles possibly caused higher and more irregular patterns on surface wear. Irregularities caused by AOS were also found on surfaces of extracted human teeth (15). In the present study, polishing possibly regulated surfaces with fragment fractures formed by AOS. 

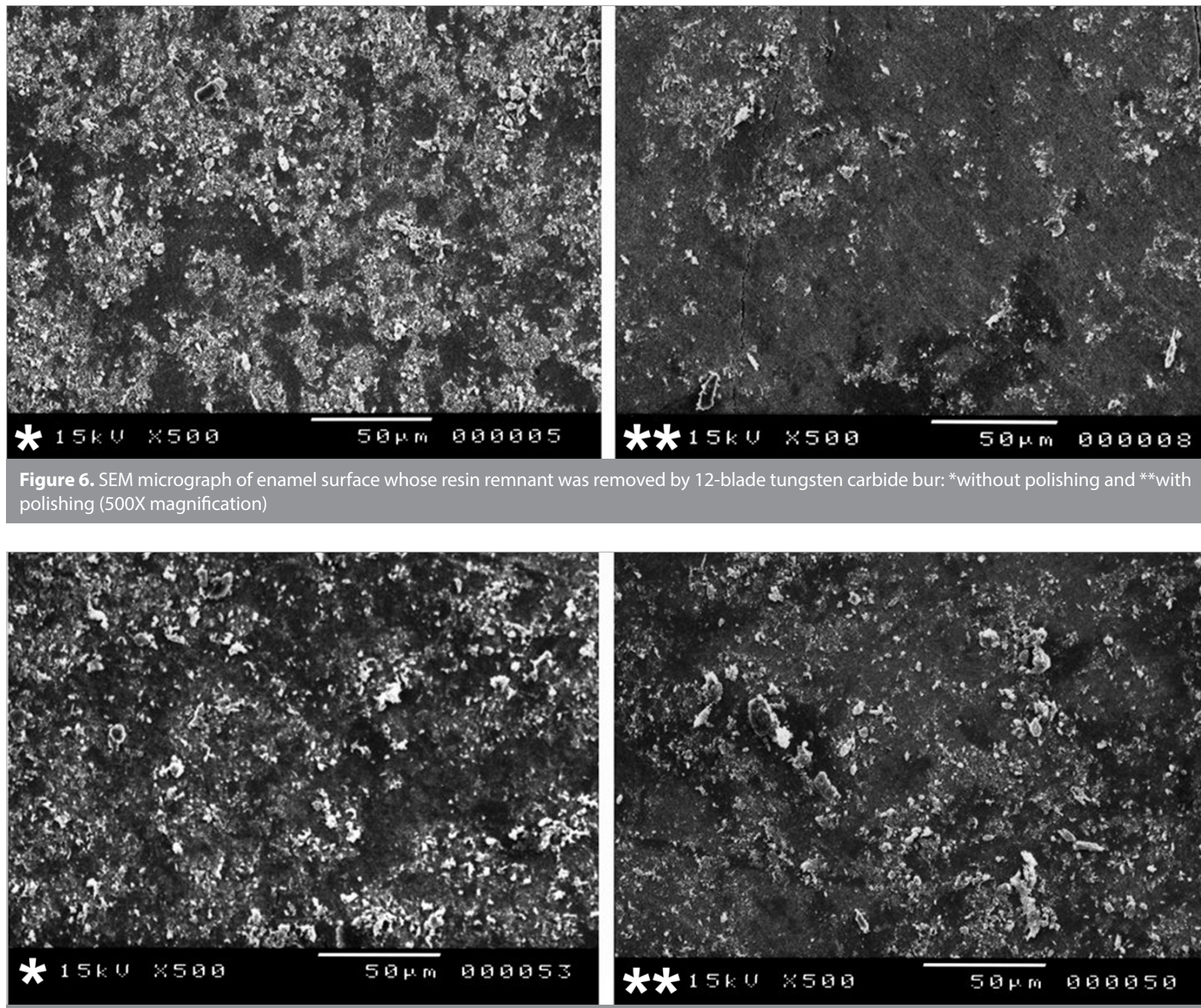

Figure 7. SEM micrograph of enamel surface whose resin remnant was removed by 6-blade tungsten carbide bur: *without polishing and **with polishing (500X magnification)

Table 5. Surface roughness means difference and 95\% confidence interval between techniques among polished and non-polished

\begin{tabular}{|c|c|c|c|c|c|c|}
\hline & & \multicolumn{5}{|c|}{ Polishing } \\
\hline & & Blade-30 & Blade-12 & Blade- 6 & AOS & Laser \\
\hline \multirow[t]{3}{*}{ No polishing } & Blade-30 & 1 & $0.10(-0.49$ to 0.70$)$ & $0.16(-0.43$ to 0.76$)$ & $1.00 *(0.41$ to 1.61$)$ & $1.82^{*}$ (1.22 to 2.42$)$ \\
\hline & Blade-6 & -0.31 (-0.91 to 0.28$)$ & $-0.31(-0.91$ to 0.28$)$ & 1 & $0.84^{*}(0.25$ to 1.44$)$ & $1.66^{*}$ (1.16 to 2.25$)$ \\
\hline & AOS & $-0.84^{*}(-1.45$ to -0.24$)$ & $-0.84 *(-1.45$ to -0.24$)$ & $-0.52(-1.2$ to 0.07$)$ & 1 & $0.81^{*}(0.21$ to 1.41$)$ \\
\hline
\end{tabular}

*Statistically different means $(p<0.05)$ for multiple comparison $t$

When comparing RRR techniques, no significant difference was found among multibladed burs, regardless of whether final polishing was performed. Among multiblade burs, 6-bladed burs showed more unsatisfactory performance. It can be verified from the dispersion of results obtained with the 6-bladed bur when compared with 12- and 30-bladed burs and the quality of the enamel surface showed by SEM. Ulusoy (24) observed that
12- and 30-fluted tungsten carbide burs at high speed with water coolant are fast and efficient in residual resin removal, but the resultant enamel surface with enamel scars needs to be finished by other polishing techniques. Although this investigation showed lower surface alterations, scratches on the surface were found when a 30-bladed bur was used, even when final polishing was performed. 

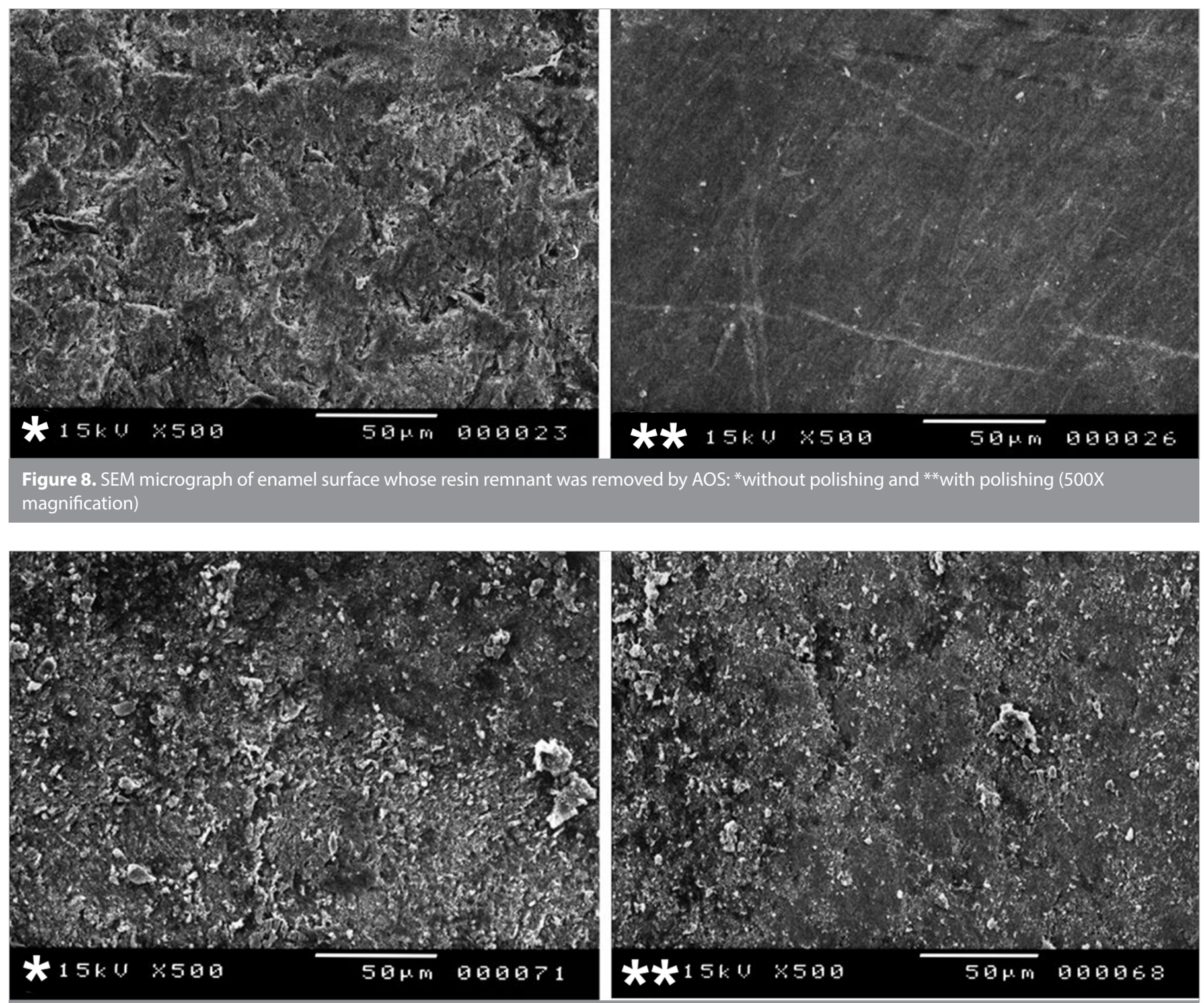

Figure 9. SEM micrograph of enamel surface whose resin remnant was removed by Er:YAG laser: * without polishing and ${ }^{* *}$ with polishing (500X magnification)

Considering AOS and laser, the test specimens on which AOS was used showed the least favorable results among the performed techniques, as greater wear depth in micrometers was found in the profilometry. However, considering the condition in which the final polishing was not performed, AOS was similar to laser because the depths recorded in both techniques were similar. This likely occurred because of the laser beam being directed, in contrast to the jet, which was applied in a dispersed manner. The intensity used in the irradiation and concentration of this beam in a smaller area than that obtained with sandblasting (jet dispersion and particle thickness) is possibly responsible for a smaller depth of surface changes than observed with the laser technique. Both sandblasting and laser were statistically different from the three multiblade tips, corroborating the study by Almeida et al. (17).

However, provided that the final polishing was performed, results for laser were similar to those of 6- and 12-bladed burs, but statistically different from abrasion and 30-bladed burs. Final polishing was important for all techniques, although the laser technique caused a decrease in the surface depth in micrometers, probably because of fractures or irregularities left during its application, leading to a smaller dispersion of the results obtained with polishing. This result is in agreement with Howell and Weekes, who affirmed that polishing performed after RRR might cause loss of the enamel. This loss is proportional to the time spent on the procedure (25).

In the present study, significant surface alterations were caused by the laser, as shown by the depths recorded by the profilometer and SEM, according to Ahrari et al. (8) and Kwon et al. (19).

When analyzing the surface roughness data, the mean values obtained by all of the techniques were higher than that recorded for the control region, corroborating the study by Kim et al. (22), who compared the RRR with low-speed tungsten bur and AOS. 
In the present study, both multiblade burs and AOS did not show significant differences.

No statistically significant difference was observed between multiblade burs for the different techniques used for removing resin remnants under conditions in which the final polishing was or was not performed.

Comparing all multiblade burs with the other techniques, only the 6-bladed bur without polishing was statistically different from the control and similar to AOS. The other results of AOS and laser were statistically significant, thus showing more damage to the enamel surface. Comparing the condition in which the final polishing was performed, the 6-, 12-, and 30-bladed burs were equal to each other and the control among all the techniques used. AOS and laser were statistically different from the control and all multiblade burs.

Among all the investigated techniques, the 30-bladed bur showed fewer surface alterations. This information is in disagreement with the study by Degrazia et al. (28). Despite improving surface quality, final polishing does not remove deep scratches, as previously reported (5). It is important to point out that it made no difference whether or not polishing was performed when using different multiblade burs.

Comparing the results from profilometry and surface roughness, it was observed that the 6-bladed bur showed higher dispersion of results, both with and without final polishing, although the 6-bladed bur did not show a significant difference from the results of the other two multiblade burs. Regarding surface roughness, 6-, 12-, and 30-bladed burs showed a more regular surface, with no statistical difference among them, although the results of the 30-bladed bur left the surface more similar to the control region.

The literature has shown that multiblade burs cause the least amount of harm to the enamel and are the most indicated for RRR, which was also found in the present study $(11,12,14,23,28)$.

Regarding AOS, although the profilometry analysis showed a higher depth and dispersion of results, the surface roughness analysis showed a less rough surface than that of the laser. The laser showed a lower depth in the profilometry analysis than in the AOS. However, surface quality observed in the roughness evaluation was comparatively less favorable when using laser than that found for AOS.

In extrapolating from the clinical orthodontics, among all the used techniques, AOS is the one that caused more enamel wear, whereas Er:YAG laser caused more irregularities, which favored the accumulation of biofilm.

All of the used techniques caused superficial irregularities and enamel loss, which could not be quantified. Thus, enamel thickness was not quantified in the evaluated surfaces. This evaluation could have shown how much of the enamel was lost, in addition to comparing the experimental areas with the control areas. Only two randomly selected teeth from each group were evaluated by SEM. The results would be more consistent if all the teeth had been subjected to electron microscopy. An in situ study will likely bring greater applicability to the clinical practice. It was, in fact, a limitation of the present study.

\section{CONCLUSION}

- The null hypothesis was rejected.

- Multiblade burs promoted fewer irregularities in the enamel, as they were less harmful than the other techniques, regardless of whether the final polishing was performed or not.

- AOS caused greater wear on the enamel surface, whereas the Er:YAG laser caused the largest irregularities on the enamel surface.

Ethics Committee Approval: Ethics Committee on Animal Use - CEUA/ FORP is responsible "for complying with and enforcing, within the scope of its attributions, the provisions of national legislation and other applicable rules to the use of live animals for teaching and research at FORP/ USP" (emphasis added), in accordance with the provisions of Art. 3 Item III and Art. 10 of Law 11,794, of 10/08/2008, not analyzing, therefore, experimental protocols that do not involve the use of live animals.

\section{Informed Consent: N/A.}

Peer-review: Externally peer-reviewed.

Author Contributions: Concept - F.L.R., M.C.B., M.A.N.M; Design - F.L.R., J.T.L.F., M.C.B., M.C.P.S.; Supervision - C.P.T., F.L.R., J.T.L.F., M.A.N.M.; Data Collection and/or Processing - J.T.L.F., M.C.P.S.; Analysus and/or Interpretation - J.T.L.F., M.C.P.S.; Writing Manuscript - J.T.L.F; Critical Review - F.L.R, M.A.N.M; Literature Search - J.T.L.F., C.P.T.

Conflict of Interest: The authors have no conflict of interest to declare.

Financial Disclosure: The authors declared that this study has received no financial support.

\section{REFERENCES}

1. Buonocore M. A simple method of increasing the adhesion of acrylic filing materials to enamel surfaces. J Dent Res 1955; 34: 849-53. [CrossRef]

2. Newman GV. Bonding plastic orthodontic attachments. J Pract Orthod 1969; 3: 231-8.

3. Zachrisson BU, Arthun J. Enamel surface appearance after various debonding techniques. Am J Orthod 1979; 75: 121-7. [CrossRef]

4. Artun J, Bergland S. Clinical trials with crystal growth conditioning as an alternative to acid-etch enamel pretreatment. Am J Orthod 1984; 85: 333-40. [CrossRef]

5. Eliades T, Gioka C, Eliades G, Makou M. Enamel surface roughness following debonding using two resin grinding methods. Eur J Orthod 2004; 26: 333-8. [CrossRef]

6. Pont HB, Özcan M, Bagis B, Ren Y. Loss of surface enamel after bracket debonding: an in-vivo and ex-vivo evaluation. Am J Orthod Dentofacial Orthop 2010; 138: 387-9. [CrossRef]

7. Ryf S, Flury S, Palaniappan S, Lussi A, Van Meerbeek B, Zimmerli B. Enamel loss and adhesive remnants following bracket removal and various clean-up procedures in vitro. Eur J Orthod 2012; 34: 25-32. [CrossRef] 
8. Ahrari F, Akbari M, Akbari J, Dabiri G. Enamel surface roughness after debonding of orthodontic brackets and various clean-up techniques. J Dent 2013; 10: 82-93.

9. Salehi P, Pakshir H, Naseri N, Baherimoghaddam T. The effects of composite resin types and debonding pliers on the amount of adhesive remnants and enamel damages: a stereomicroscopic evaluation. J Dent Res Dent Clin Dent Prospects 2013; 7: 199-205.

10. Webb BJ, Koch J, Hagan JL, Ballard RW, Armbruster PC. Enamel surface roughness of preferred debonding and polishing protocols. J Orthod 2016; 43: 39-46. [CrossRef]

11. Mohebi S, Shafiee HA, Ameli N. Evaluation of enamel surface roughness after orthodontic bracket debonding with atomic force microscopy. Am J Orthod Dentofacial Orthop 2017; 151: 521-7. [CrossRef]

12. Ferreira FG, Nouer DF, Silva NP, Garbui IU, Correr-Sobrinho L, Nouer PR. Qualitative and quantitative evaluation of human dental enamel after bracket debonding: a non-contact three-dimensional optical profilometry analysis. Clin Oral Investig 2014; 18: 1853-64. [CrossRef]

13. Vidor MM, Felix RP, Marchioro EM, Hahn L. Enamel surface evaluation after bracket debonding and different resin removal methods. Dental Press J Orthod 2015; 20: 61-7. [CrossRef]

14. Cardoso LA, Valdrighi HC, Vedovello Filho M, Correr AB. Effect of adhesive remnant removal on enamel topography after bracket debonding. Dental Press J Orthod 2014; 19: 105-12. [CrossRef]

15. Sargison AE, McCabe JF, Millett DT. A laboratory investigation to compare enamel preparation by sandblasting or acid etching prior to bracket bonding. Br J Orthod 1999; 26: 141-6. [CrossRef]

16. Mhatre AC, Tandur AP, Reddy SS, Karunakara BC, Baswaraj H. Enamel surface evaluation after removal of orthodontic composite remnants by intraoral sandblasting technique and carbide bur technique: a three-dimensional surface profilometry and scanning electron microscopic study. J Int Oral Health 2015; 7: 34-9.

17. Almeida HC, Vedovello Filho M, Vedovello SA, Young AA, RamirezYañez GO. ER:YAG laser for composite removal after bracket debonding: a qualitative SEM analysis. Int J Orthod Milwaukee 2009; 20: 9-13.
18. Macri RT, de Lima FA, Bachmann L, Galo R, Romano FL, Borsatto MC, et al. $\mathrm{CO} 2$ laser as auxiliary in the debonding of ceramic brackets. Lasers Med Sci 2015; 30: 1835-41. [CrossRef]

19. Kwon $\mathrm{YH}, \mathrm{Kwon} \mathrm{OW}, \mathrm{Kim} \mathrm{HI}$, Kim KH. Nd:YAG laser ablation of enamel for orthodontic use: tensile bond strength and surface modification. Dent Mater J 2003; 22: 397-403. [CrossRef]

20. Pus MD, Way DC. Enamel loss due to orthodontic bonding with filled and unfilled resins using various clean-up techniques. Am J Orthod 1980; 77: 269-83. [CrossRef]

21. Thompson RE, Way DC. Enamel loss due to prophylaxis and multiple bonding/debonding of orthodontic attachments. Am J Orthod 1981; 79: 282-95. [CrossRef]

22. Kim SS, Park WK, Son WS, Ahn HS, Ro JH, Kim YD. Enamel surface evaluation after removal of orthodontic composite remnants by intraoral sandblasting: a 3-dimensional surface profilometry study. Am J Orthod Dentofacial Orthop 2007; 132: 71-6. [CrossRef]

23. Koprowski R, Machoy M, Woźniak K, Wróbel Z. Automatic method of analysis of OCT images in the assessment of the tooth enamel surface after orthodontic treatment with fixed braces. Biomed Eng Online 2014; 13: 48. doi: 10.1186/1475-925X-13-48. [CrossRef]

24. Ulusoy $C$. Comparison of finishing and polishing systems for residual resin removal after debonding. J Appl Oral Sci 2009; 17: 209-15. [CrossRef]

25. Howell S, Weekes WT. An electron microscopic evaluation of the enamel surface subsequent to various debonding procedures. Aust Dent J 1990; 35: 245-52. [CrossRef]

26. Janiszewska-Olszowska J, Szatkiewicz T, Tomkowski R, Tandecka K, Grocholewicz K. Effect of orthodontic debonding and adhesive removal on the enamel - current knowledge and future perspectives a systematic review. Med Sci Monit 2014; 20: 1991-2001. [CrossRef]

27. Sfondrini MF, Scribante A, Fraticelli D, Roncallo S, Gandini P. Epidemiological survey of different clinical techniques of orthodontic bracket debonding and enamel polishing. J Orthod Sci 2015; 4: 123 7. [CrossRef]

28. Degrazia FW, Genari B, Ferrazzo VA, Santos-Pinto AD, Grehs RA. Enamel Roughness Changes after Removal of Orthodontic Adhesive. Dent J (Basel) 2018; 6: pii: E39. doi: 10.3390/dj6030039. [CrossRef] 